\title{
Significance of Controlling Crystallization Mechanisms and Kinetics in Pharmaceutical Systems
}

\author{
Naír Rodríguez-Hornedo ${ }^{*, \dagger}$ and Denette Murphy ${ }^{\ddagger}$
}

Contribution from College of Pharmacy, University of Michigan, Ann Arbor, Michigan 48109-1065 and Dupont Pharmaceuticals, P.O. Box 80400, Wilmington, Delaware 19880-0400.

Received December 23, 1998. Final revised manuscript received April 8, 1999. Accepted for publication April 12, 1999.

\section{Introduction}

Metastable thermodynamic states are frequently encountered in pharmaceutical systems, in the intentional or unintentional creation of supersaturation, in the crystallization of desired solid-state modifications, and in the control of solid-phase conversions during isolation, manufacturing, storage, and dissolution. ${ }^{1-4}$ Some examples in which metastable states are encountered indude solid solutions, freeze-concentrated solutions, solutions of weak acids or bases exposed to a $\mathrm{pH}$ change, solutions prepared by dissolving a solid-state modification with a higher solubility (higher free energy), and residual solutions during filtration, granulation, and drying. Because crystallization provides a way of reducing the free energy of metastable thermodynamic states, the extent to which metastable states can be maintained is determined by the crystallization mechanisms and kinetics. ${ }^{5-16}$ What is surprising, however, is that despite the important role that crystallization has in process control and in determining solid-phase outcomes, crystallization phenomena are often neglected in the pharmaceutical industry until a problem is encountered.

While emphasis is often given to the knowledge of equilibrium phase diagrams with the purpose of identifying the concentration and temperature regions of thermodynamic stability of solid phases, information on crystallization processes can only be obtained by combining studies

* Corresponding author. Tel: 734-763-0101. Fax: 734-763-2022. e-mail: nrh@umich.edu.

† University of Michigan.

₹ Dupont Pharmaceuticals. of thermodynamic properties with kinetic measurements. Cases of unwanted or previously unknown nucleation events abound. Dunitz and Bernstei ${ }^{17}$ documented cases of "disappearing or elusive polymorphs" that provide evidence for the consequences of poor process control in crystallization of polymorphic systems. The recent shortage in the supply of capsules of the HIV protease inhibitor Norvir (indinavir), due to the sudden formation of a crystalline structure different from the one harvested for months, ${ }^{18}$ illustrates the decisive role that nucleation mechanisms and kinetics have on crystallization. Nichols and Frampton ${ }^{19}$ have reported considerable efforts that failed to crystallize the metastable polymorph of paracetamol as described in the initial publication of the crystal structure. ${ }^{20}$ The critical role of crystallization kinetics in determining the appearance of crystalline modifications is also recognized by the FDA and described in the guidel ines for the manufacture of drug substances:21 "Appropriate manufacturing and control procedures (including in-process testing when needed) should be established for the production of the desired solid-state form(s). It should be emphasized that the manufacturing process (or storage condition) is responsible for producing particular polymorphs or solvates; the control methods merely determine the outcome."

Even when the parameters that regulate crystallization phenomena are neglected, the illusion of process control is motivated by a crystallization process that yields the desired product-solid phase modification, shape, or size distribution-and by the robust analytical methods used for solid state characterization. This situation is greatly complicated by the recent emphasis on an exclusively 
structural approach due to the availability of commercial software for the prediction of crystal structure from molecular structure (ab initio predictions) while kinetic processes are scarcely investigated. Desiraju22-24 and Gave zzotti ${ }^{25-29}$ have discussed the various methods for crystal structure and polymorphism prediction and the capabilities and shortcomings of the various computer simulation techniques. The main challenge lies in determining the relationships between the molecular aggregation pathways that cause some nuclei to grow and the structure and thermodynamics of the crystalline solids. Recent work from various groups ${ }^{14,30-40}$ highlight the importance of combining computer simulations with experimental methods to investigate the mol ecular events that lead to crystallization.

The purpose of this review is to describe ways in which crystal structure, morphology, and crystallization kinetics can be utilized to reproducibly maintain metastable states and control solid-state outcomes. Experimental methods that can be employed to investigate the factors that regulate crystallization from solution will be presented.

\section{Crystallization}

A crystalline phase is created as a consequence of molecular aggregation processes in solution that lead to the formation of nuclei (with a configuration compatible with the crystal structure), which achieve a certain size for a sufficient time to enable growth into macroscopic crystals. The rate and mechanisms by which crystals are formed in liquid solutions is determined by the solubility, supersaturation, rate at which supersaturation is created, diffusivity, temperature, and the reactivity of surfaces toward nucleation. ${ }^{41-45}$

Transferability of crystallization microtechniques to larger scale processes that are reproducible requires identification of crystallization mechanisms and kinetics. While operationally useful variables that describe crystallization methods are often related to crystallization outcomes, this approach lacks meaningful information for developing a process that yiel ds reliable outcomes, since the factors that determine the crystallization kinetics and outcomes are not explicitly considered. For instance, compare the following two approaches to describe the processes for the selective crystallization of polymorphs: (1) form I obtained by cooling, form II obtained by evaporation, and (2) form I obtained at a supersaturation $x$, temperature $y$, and time $z$ at which crystals were harvested after crystallization onset; form II obtained at supersaturation $x^{\prime}$, etc. While the former approach is at best anecdotal, the latter employs the causative factors and leads to relationships between the crystallization kinetic parameters and the outcomes.

Control of the processes of nucleation and crystal growth is possible as long as the required information is available. Dunitz and Bernstein ${ }^{17}$ have explained the mystery of disappearing polymorphs by considering various examples and the relevant questions that were left unanswered. They state that "once a particular polymorph has been obtained, it is always possible to obtain it again; it is only a matter of finding the right experimental conditions."

\section{Supersaturation}

Knowledge of the driving force for crystallization is essential, not only to characterize the kinetics, but also to relate the crystallization outcomes to the parameters that regulate crystallization. The number of molecules necessary to achieve an effective nucleating cluster is inversely proportional to the supersaturation. Therefore, as the supersaturation is increased the probability of nucleation increases. However, nucleation is energetically more demanding than crystal growth, and there are supersaturation regions in which crystal growth proceeds while nucleation is suppressed. $41,42,44$

The driving force for nucleation and growth is the difference in chemical potential of the solute in a supersaturated solution, $\mu_{1}$, and in a saturated solution, $\mu_{\text {eq: }}$

$$
\Delta \mu=\left(\mu_{1}-\mu_{\mathrm{eq}}\right)
$$

Since $\mu=\mu^{\circ}+\mathrm{RT}$ In $\mathrm{a}$, then

$$
\Delta \mu=\mathrm{RT} \ln \left(\mathrm{a}_{1} / \mathrm{a}_{\mathrm{eq}}\right)=\mathrm{RT} \ln \left(\gamma_{1} \mathrm{C}_{1} / \gamma_{\mathrm{eq}} \mathrm{C}_{\mathrm{eq}}\right)
$$

and the supersaturation is

$$
\sigma=\Delta \mu / \mathrm{RT}=\ln \left(\mathrm{a}_{1} / \mathrm{a}_{\mathrm{eq}}\right)=\ln \left(\gamma_{1} \mathrm{C}_{1} / \gamma_{\mathrm{eq}} \mathrm{C}_{\mathrm{eq}}\right)
$$

If the activity coefficient, $\gamma$, is independent of concentration, in the given concentration regime, then $\gamma_{1}=\gamma_{\text {eq }}$ and the supersaturation becomes

$$
\sigma=\ln \left(\mathrm{c}_{1} / \mathrm{c}_{\mathrm{eq}}\right)=\ln (\mathrm{c} / \mathrm{s})
$$

where $c$ is the concentration of the crystallizing substance in the supersaturated solution and s is the solubility. (This notation is adopted to avoid the use of subscripts.) A frequently used definition for the supersaturation is

$$
\sigma \equiv(\mathrm{c}-\mathrm{s}) / \mathrm{s}
$$

For c/s values smaller than 1.15,

$$
\sigma=\ln (\mathrm{c} / \mathrm{s}) \approx(\mathrm{c}-\mathrm{s}) / \mathrm{s}
$$

Supersaturation may be created by various methods that regulate the solute activity (concentration) or activity product. These include (a) solvent removal (evaporation or freezing), (b) addition of indifferent salts with ions that participate in precipitation, and (c) dissolution of metastable solid phases. Supersaturation can also be created by methods that regulate the solute solubility, such as temperature change, $\mathrm{pH}$ change, and addition of a solvent that lowers the solubility of the solute.

\section{Nucleation}

Nucleation is often the decisive step in the crystallization process and is of practical importance in pharmaceutical systems. For instance, questions regarding the concentration threshold above which crystallization is observed at times shorter than the desired product shelf life, or dilution rates and concentrations above which precipitation occurs upon injection, are related to the kinetic stability of supersaturated solutions and are regulated by the nucleation mechanisms and kinetics. Nucl eation phenomena are equally important in the control of micromeritic properties and in the selective crystallization of a particular polymorph.

In general, nucleation mechanisms can be divided into two main categories: homogeneous and surface or interface catalyzed. ${ }^{42-44,46} \mathrm{H}$ omogeneous nucleation rarely occurs in large volumes (greater than $100 \mu \mathrm{l}$ ) since sol utions contain random impurities which may induce nucleation. 47,48 This type of nucleation is referred to as heterogeneous. A surface or an interface of different composition than the crystallizing solute may serve as a nucleation substrate, by decreasing the energy barrier for the formation of nudei that can grow into mature crystals. Nucleation that is promoted by crystals of the crystallizing solute is known 
as secondary nucl eation. These mechanisms are thoroughly discussed by Mullin, ${ }^{42}$ Myerson, ${ }^{44}$ and Zettlemoyer. ${ }^{46}$

Homogeneous Nucleation-Thermodynamic considerations for nucleation are based on the work of Gibbs, 49 Volmer ${ }^{50}$ and others, where the free energy change for an aggregate undergoing a phase transition $\Delta \mathrm{G}$ is given by

$$
\Delta G=\Delta G_{v}+\Delta G_{s}
$$

where $\Delta G_{s}$ is the surface free energy change associated with the formation of the aggregate (a positive quantity), and $\Delta \mathrm{G}_{\mathrm{v}}$ is the volume free energy change associated with the phase transition (a negative quantity). For homoge neous or heterogeneous nucleation

$$
\Delta \mathrm{G}_{\mathrm{v}}=-\alpha \mathrm{l}^{3} v \mathrm{k}_{\mathrm{B}} \mathrm{T} \ln \left(\frac{\mathrm{C}}{\mathrm{s}}\right)
$$

where $\alpha$ is the volume-shape factor, I is the characteristic length, $v$ is the mol ecular vol ume of the crystallizing sol ute, $\mathrm{k}_{\mathrm{B}}$ is Boltzmann's constant, and $\mathrm{T}$ is temperature. For homogeneous nucleation

$$
\Delta \mathrm{G}_{\mathrm{s}}=\left.\beta\right|^{2} \gamma_{12}
$$

where $\beta$ is the area shape factor and $\gamma$ is the interfacial energy per unit area between the crystallization medium, 1 , and the nucleating cluster, 2 . Consequently, the overall free energy change for nucleation is decreased by a large supersaturation ratio (c/s) and by a low interfacial energy.

The factors that regulate nucl eation are best appreciated by considering the equation for the rate of homogeneous nucleation from solutions:

$$
J=\mathrm{N}_{0} v \exp \left(\frac{-\Delta \mathrm{G}^{*}}{\mathrm{k}_{\mathrm{B}} \mathrm{T}}\right)=\mathrm{N}_{\mathrm{o}} v \exp \left(\frac{-4 \beta^{3} v^{2} \gamma_{12}^{3}}{27 \alpha^{2}\left(\mathrm{k}_{\mathrm{B}} \mathrm{T}\right)^{3}\left(\ln \left(\frac{\mathrm{c}}{\mathrm{s}}\right)\right)^{2}}\right)
$$

$J$ is the number of nuclei formed per unit time per unit volume, $\mathrm{N}_{\mathrm{o}}$ is the number of molecules of the crystallizing phase in a unit volume, $v$ is the frequency of atomic or molecular transport at the nucleus-liquid interface, and $\Delta \mathrm{G} *$ is the maximum in the Gibbs free energy change for the formation of clusters, at a certain critical size, I*. The nucleation rate was initially derived for condensation in vapors by Becker and Döring, ${ }^{51}$ where the preexponential factor is related to the gas kinetic collision frequency. In the case of nucleation from condensed phases, the fre quency factor is related to the diffusion process. ${ }^{52}$ The value of I* can be obtained by minimizing the free energy function with respect to the characteristic length

$$
\mathrm{I}=\frac{2 \beta v \gamma_{12}}{3 \alpha \mathrm{k}_{\mathrm{B}} \mathrm{T} \ln \left(\frac{\mathrm{c}}{\mathrm{s}}\right)}
$$

For spherical clusters, $\alpha=4 \pi / 3$ and $\beta=4 \pi$ based on the cluster radius. Therefore,

$$
\mathrm{r}^{*}=\frac{2 v \gamma_{12}}{\mathrm{k}_{\mathrm{B}} \mathrm{T} \ln \left(\frac{\mathrm{c}}{\mathrm{s}}\right)}
$$

Considering these geometric factors, the rate for homogeneous nucleation of spherical clusters is

$$
J=\mathrm{N}_{0} v \exp \left(\frac{-16 \pi v^{2} \gamma_{12}^{3}}{3\left(\mathrm{k}_{\mathrm{B}} \mathrm{T}\right)^{3}\left(\ln \left(\frac{\mathrm{c}}{\mathrm{s}}\right)\right)^{2}}\right)
$$

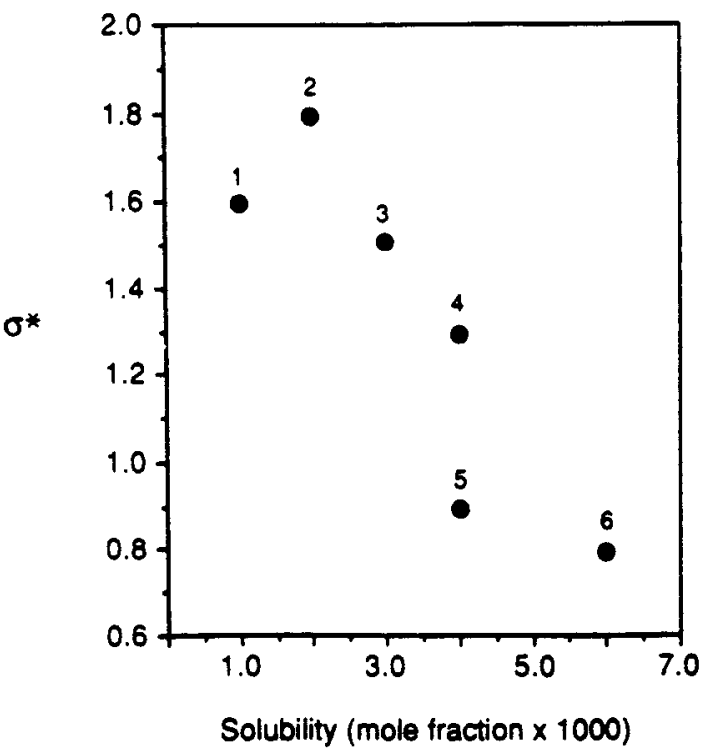

Figure 1-Dependence of the critical supersaturation for nucleation on solubility of nitrofurantoin in (1) formic acid, (2) formic acid:water (4:1), (3) formic acid: ethanol (2:1), (4) formic acid:dioxane (2:1), (5) formic acid:methanol (2:1), (6) formic acid:water (2:1). Data from ref 55 .

While the classical theory of nucleation is limited by the implicit assumptions in its derivation (described in detail in ref 41) it successfully predicts the nudeation behavior of a system. ${ }^{42-44,46,53}$ Inspection of the equation above clearly suggests that the nucleation rate can be experimentally controlled by the following parameters: molecular or ionic transport, viscosity, supersaturation, solubility, solid-liquid interfacial tension, and temperature

Nucleation kinetics are experimentally determined from measurements of nucleation rates, induction times and metastability zone widths (the supersaturation or undercooling necessary for spontaneous nucleation) as a function of initial supersaturation. ${ }^{41-43,54}$ The nudeation rate will increase by increasing the supersaturation while all other variables are constant. However, at constant supersaturation the nucleation rate will increase with increasing solubility. Solubility affects the pre-exponential factor and the probability of intermolecular collisions. F urthermore, when changes in solvent or solution composition lead to increases in solubility, the interfacial energy decreases since the affinity between crystallizing medium and crystal increases. ${ }^{41}$ Consequently, the supersaturation required for spontaneous nucleation decreases with increasing solubility, 55 as shown in Figure 1.

The dependence of nudeation rate on solubility is also consistent with Ostwald's law of stages ${ }^{56}$ regarding the preferential formation of a metastable solid phase, that states the following: "when leaving an unstable state, a system does not seek out the most stable state, rather the nearest metastable state which can be reached with loss of free energy". This indicates that if the unstable solidstate modification (the system with highest solubility) precipitates before more thermodinamically stable solid phases, it must have higher nucleation and growth rates than solid states of lower solubility. However, Ostwald's law of stages is not universally valid because the appearance and evolution of solid phases are determined by the kinetics of nucleation and growth under the specific experimental conditions. ${ }^{5,6,13}$

Accounts of nucleation inhibition in the pharmaceutical literature are sometimes confusing because the dependence of the nucleation event (nucleation rate, metastability zone width, or induction time) on supersaturation is not considered. In search for additives that inhibit nucleation, 
Table 1-Comparison of Crystallization Behavior of Disodium Phosphate during Far-from-Equilibrium Freezing of Buffer Solutions

\begin{tabular}{|c|c|c|}
\hline \multicolumn{2}{|c|}{ crystallization behavior of $\mathrm{Na}_{2} \mathrm{HPO}_{4} \cdot 12 \mathrm{H}_{2} \mathrm{O}$} & \multirow{2}{*}{$\begin{array}{l}\mathrm{C}_{\mathrm{Na}_{2} \mathrm{HPO}}{ }_{4}{ }^{a} \\
(\mathrm{mM})\end{array}$} \\
\hline reference & observation & \\
\hline \multirow[t]{2}{*}{ Murase and Franks $\mathrm{s}^{63,64}$} & $\begin{array}{l}\text { crystallization begins to } \\
\text { decrease }\end{array}$ & $\leq 200$ \\
\hline & does not crystallize & $\leq 10$ \\
\hline \multirow{2}{*}{$\begin{array}{l}\text { Cavatur and } \\
\quad \text { Suryanarayanan }{ }^{65}\end{array}$} & readily crystallizes & 190 \\
\hline & $\begin{array}{l}\text { crystallization is inhibited } \\
\text { by } C_{\mathrm{NaH}_{2} \mathrm{PO}_{4}{ }^{b} \geq 730 \mathrm{mM}}\end{array}$ & 190 \\
\hline \multirow[t]{3}{*}{ Gómez $z^{66}$} & $\begin{array}{l}\text { crystallization begins to } \\
\text { decrease }\end{array}$ & \\
\hline & crystallization readily occurs & $\geq 0.3$ \\
\hline & $\begin{array}{l}\text { crystallization is not inhibited } \\
\text { at } C_{\mathrm{NaH}_{2} \mathrm{PO}_{4}} \leq 94 \mathrm{mM}\end{array}$ & 6 \\
\hline
\end{tabular}

${ }^{a} \mathrm{C}_{\mathrm{Na}_{2} \mathrm{HPO}_{4}}=$ initial disodium phosphate concentration in solution at $25^{\circ} \mathrm{C}$. ${ }^{b} \mathrm{C}_{\mathrm{NaH}_{2} \mathrm{PO}_{4}}=$ initial monosodium phosphate concentration in solution at $25^{\circ} \mathrm{C}$.

induction times are often measured as a function of additive concentration while the dependence of the nucle ation event on supersaturation is neglected. Results from such studies possibly lead to the erroneous conclusion that the additive inhibited nucleation ${ }^{57,58}$ when indeed the additive decreased the supersaturation, and frequently led to an undersaturated state. Hence, the system is under thermodynamic control instead of kinetic control.

An important factor contributing to the nudeation mechanism and kinetics is the volume of solution in which nucleation occurs. The dispersal of a bulk liquid into a collection of small droplets has been shown to be an effective way of achieving large supersaturations or undercoolings. ${ }^{47,48}$ Precipitation and solidification in small volumes (droplets) involving emulsions have been used to study homogeneous nucleation processes, ${ }^{59}$ and to control purity, particle size, and morphology. ${ }^{60,61}$ Dispersing a solution into small volumes isolates heterogeneous nucleants within a fraction of the drops and makes nucleation more difficult. Consequently, larger supersaturations need to be reached for nucleation to occur. The boundaries of possible outcomes are represented by the following sce narios: (1) crystals of very small size (even in the nanometer range) are formed as a result of the high nucleation rates, ${ }^{60,61}$ or (2) a glass or amorphous solid is formed due to the low diffusion rates of molecules that inhibit the evolution of clusters to crystals within the time scale of the experiment. ${ }^{62}$

Nucleation outcomes from solutions with initially the same composition may vary as a consequence of impurities, rates at which supersaturation was created, thermal histories, experimental techniques employed to detect precipitation, and solution volumes in which nucleation occurred. An important factor contributing to the changes in $\mathrm{pH}$ during freezing of buffer solutions is the selective crystallization of buffer components. ${ }^{63-66}$ The salt and buffer concentrations for precipitation of disodium phosphate during freezing of sodium phosphate buffer solutions among various laboratories are shown in Table 1. Murase et al. ${ }^{63,64}$ report higher initial buffer and disodium phosphate concentrations for the precipitation of disodium phosphate, compared to results from our laboratory. ${ }^{66}$ The fraction of disodium phosphate precipitated was observed to decrease with decreasing the initial buffer concentration below $500 \mathrm{mM}$, at $200 \mathrm{mM}$ disodium phosphate concentration, versus an initial buffer concentration below $8 \mathrm{mM}$, at $0.3 \mathrm{mM}$ disodium phosphate concentration in our studies; ${ }^{66}$ furthermore, disodium phosphate did not precipitate at initial disodium salt concentrations below $10 \mathrm{mM} 63,64$
Table 2-Experimental Conditions and Methods of Measuring Progress of Sodium Phosphate Crystallization during Freezing

\begin{tabular}{|c|c|c|c|c|}
\hline reference & $\begin{array}{l}\text { vol of } \\
\text { solution } \\
(\mu \mathrm{L})\end{array}$ & $\begin{array}{l}\text { cooling } \\
\text { rate } \\
\left({ }^{\circ} \mathrm{C} / \mathrm{min}\right)\end{array}$ & $\begin{array}{l}\text { temp } \\
\left({ }^{\circ} \mathrm{C}\right)\end{array}$ & method \\
\hline Murase and Franks ${ }^{63,64}$ & $2-5$ & 0.62 & -52 & DSC, SEM \\
\hline $\begin{array}{l}\text { Cavatur and } \\
\text { Suryanarayanan } 65\end{array}$ & $300^{a}$ & 15 & -40 & XRPD \\
\hline Gómez $^{66}$ & $25 \times 10^{3}$ & $0.3-0.5$ & -10 & $\mathrm{pH}$ \\
\hline
\end{tabular}

a Personal communication.

while our studies show that precipitation occurs at salt concentrations as low as $0.3 \mathrm{mM} .66$ Inspection of the experimental conditions under which these studies were done (Table 2) shows a trend toward slower salt precipitation rates with decreasing volume of solutions from $25 \mathrm{~mL}$ to $3 \mu \mathrm{l}$ and increasing rates of cooling to lower temperatures.

Neglecting the factors that regulate nucleation leads to misleading generalizations when developing guidelines to control precipitation by the addition of noncrystallizing additives. Consider, for example, the conflicting interpretation of additive effects on nucleation when these are expressed in terms of concentration ratios (additive to crystallizing solute) while ignoring other parameters. Data in Table 1 indicates that disodium phosphate precipitation is inhibited at $\left(\mathrm{H}_{2} \mathrm{PO}_{4}{ }^{-} / \mathrm{HPO}_{4}{ }^{2-}\right)=4(0.73 \mathrm{M} / 0.19 \mathrm{M})^{65}$ while it is not inhibited at $\left(\mathrm{H}_{2} \mathrm{PO}_{4}^{-} / \mathrm{HPO}_{4}{ }^{2-}\right)=16(0.094$ M/0.006 M). ${ }^{66}$ Compared to the systems we studied, ${ }^{66}$ the buffers studied by Cavatur and Suryanarayana ${ }^{65}$ have much higher concentrations of monosodium phosphate (which increase the viscosity of solutions), smaller solution volumes, and faster rates of cooling to lower temperatures. All these factors contribute to delaying salt precipitation.

The effect of the viscosity of the crystallization medium on the nucleation rate has been described by Turnbull and Fisher. ${ }^{52}$ The frequency of atomic or molecular transport at the nucleus-liquid interface can be related to the bulk viscosity, $\eta$, with the Stokes-Einstein relation:

$$
v \approx \frac{\mathrm{kT}}{3 \pi \mathrm{a}_{0}^{3} \eta(\mathrm{T})}
$$

where $a_{0}$ is the mean effective diameter of the diffusing species. If the viscosity dependence on temperature is described by Arrhenius behavior, then

$$
\mathrm{J}=\frac{\mathrm{K}}{\eta_{\mathrm{o}}} \exp \left(\frac{-\Delta \mathrm{G}^{*}-\Delta \mathrm{G}_{\mathrm{a}}}{\mathrm{k}_{\mathrm{B}} \mathrm{T}}\right)
$$

where $\Delta G_{a}$ is the activation energy for transport across the nucleus-liquid interface. Thus, the nucleation rate may go through a maximum when an increase in undercooling or supersaturation is accompanied by an increase in viscosity. This behavior has been observed in the nucl eation of citric acid in aqueous solutions, ${ }^{67}$ and the crystallization of ice. 68

Heterogeneous Nucleation-Heterogeneous nucleation processes are of fundamental and practical importance in pharmaceutical systems since unintentionally or intentionally added surfaces or interfaces may promote nucleation. The reactivity of crystals surfaces as heterogeneous nucleants has significant consequences in the isolation of the desired solid-state modification and in the control of conversions between these modifications, since the free energy required for the formation of two-dimensional nuclei is lowered by the presence of an appropriate substrate. Quantitatively this is described by the following equation: 69,70 


$$
\Delta G_{s}=\gamma_{12} A_{12}+\left(\gamma_{23}-\gamma_{13}\right) A_{23}
$$

where $\gamma$ is the interaction energy per area, A is the surface area of the interfaces, and the subscript 3 represents the substrate. The total change in surface free energy will be lowered by favorable surface interactions between the aggregate and the substrate and unfavorable interactions between the crystallization medium and the substrate, due to the negative value of the second term in eq 16. Consequently, nucleation will be enhanced by increasing the surface area of the substrate.

The effectiveness of crystal seeding in controlling crystallization outcomes relies on the potential of crystal surfaces to promote heterogeneous or secondary nucleation, 42,43 while avoiding heterogeneous nucleation mediated by unknown contaminants. A review by Ward ${ }^{71}$ on the structure, properties, and reactivity of organic crystal surfaces is recommended to develop strategies for the choice of surfaces that promote nucleation. Various studies ${ }^{8,72-75}$ have demonstrated the influence of substrate topography, lattice parameters, crystallographic symmetry and intermolecular interactions on surface-directed nucleation.

Heterogeneous nud eation mechanisms can significantly affect dissolution of metastable solid phases, because this form of nucleation can occur at low driving forces. While the choice of a metastable solid phase with a solubility higher than other crystalline modifications is motivated by the expectation of faster dissolution rates, achievement of faster dissolution rates and higher concentrations in solutions is jeopardized by surface-mediated nucleation events. We have reported ${ }^{8}$ that the surface of the metastable phase of theophylline promoted the nucleation of the stable monohydrate crystals. The observed oriented growth of monohydrate crystals on the anhydrous surface is consistent with a close lattice match between the $b$ and $c$ crystallographic axes. ${ }^{8}$ Other studies on the dissolution of metastable solids such as anhydrous theophylline ${ }^{76}$ and anhydrous carbamazepine ${ }^{77-79}$ have shown that crystallization of the stable phase occurs during dissolution, Figure 2. It is unfortunate that in view of the important influence that nucleation mechanisms have on dissolution of metastable solid phases, very seldom are studies carried out to identify the potential of substrate-mediated nucleation by the metastable modification. The information gained from this type of study can be used in the design of methods to regulate crystallization during dissolution as well as during isolation of the desired solid form.

Carter and Ward ${ }^{70}$ have identified a surface-mediated nucleation mechanism that involves a geometric shape match between planes of a ledge site on the substrate and planes of prenucleation aggregates. They have applied these concepts to the directed nucleation of polymorphs. ${ }^{72,74}$ This work provides us with the attractive possibility that "a library of organic seeds can be used to control polymorphism, or to search for unknown polymorphs". ${ }^{71}$ Molecular interpretations based on this approach are experimentally more accessible than those based on solvent-selective polymorph crystallization.

Experimental and Computational Strategies-While nucleation phenomena have their origin at the molecular level, they are often described in terms of macroscopic properties due to the scarcity of experimental techniques that allow for monitoring events at the molecular level. Nevertheless, information about molecular association processes in supersaturated systems obtained by laser Raman spectroscopy and laser light scattering has been used to identify prenucleation clusters and growth units under well-defined experimental conditions. Methods that measure cluster size distributions are more appropriate for

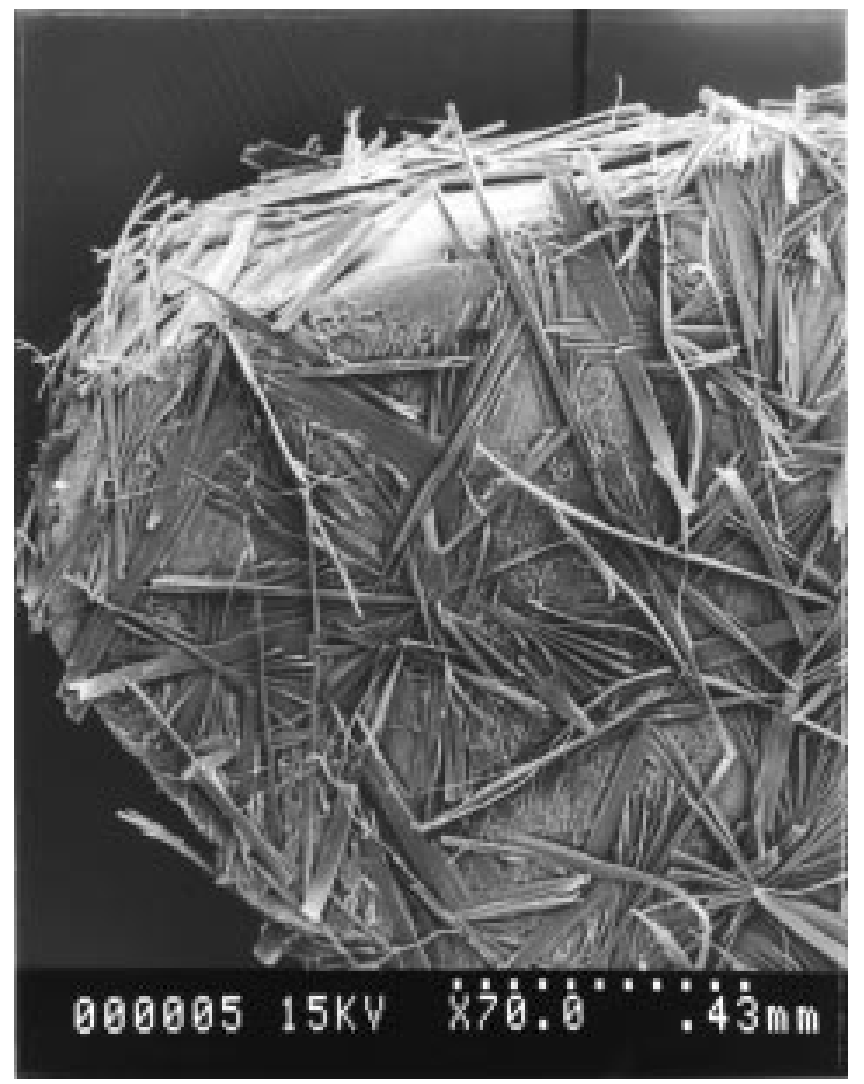

Figure 2-Nucleation and growth of dihydrate carbamazepine on crystal faces of anhydrous monoclinic carbamazepine in aqueous solutions of sodium lauryl sulfate $(17.3 \mathrm{mM}, 0.5 \%)$, supersaturation ratio of 1.15 at $25^{\circ} \mathrm{C}$. From ref 79 .

studying crystallization of macromolecules ${ }^{80-84}$ due to the large sizes of prenucleation clusters, while Raman and fluorescence spectroscopic techniques are capable of providing information about the solution structure or the species present in solution. ${ }^{85-88}$ The implications for crystallization pathways are examined by comparing the solution and crystal structures at a molecular level, and by combining information obtained from macroscopic analysis with re sults from molecular simulations. ${ }^{14,31-40}$

Desiraju $22-24$ and Gavezzotti $26-29$ have described crystal engineering strategies to understand the molecular aggregation processes involved in crystallization and to elucidate the supramolecular motifs in organic crystals. In this context, crystals are viewed as solid-state supermolecules assembled by intermolecular interactions, with the basic approach of establishing a relation between molecular interactions and supramolecular structure. Recent studies by Gavezzotti ${ }^{29}$ show how molecular dynamics calculations allow for simulation of solvent and kinetic effects on molecular aggregation.

The supramolecular assembly process can be controlled so that the precursor nudei in solution adopt a structure that resembles the structure of the desired crystalline modification. ${ }^{29,89,90}$ This concept has been used in the design of nucleation inhibitors to prevent growth of the stable polymorph and enhance the growth of the metastable polymorph. ${ }^{13,15,16}$ Davey et al. ${ }^{14}$ have explained the solventdependent polymorph appearance of sulfathiazole by analyzing the intermolecular interactions in the various polymorphic structures and comparing them with the supramole cular assemblies that could exist in the different solvents. In this case, however, the solvent-dependent selective crystallization of a polymorph was not correlated with solubility. ${ }^{14,91}$

Strategies used for the rational choice of additives to selectively inhibit nucleation or growth of the thermody- 


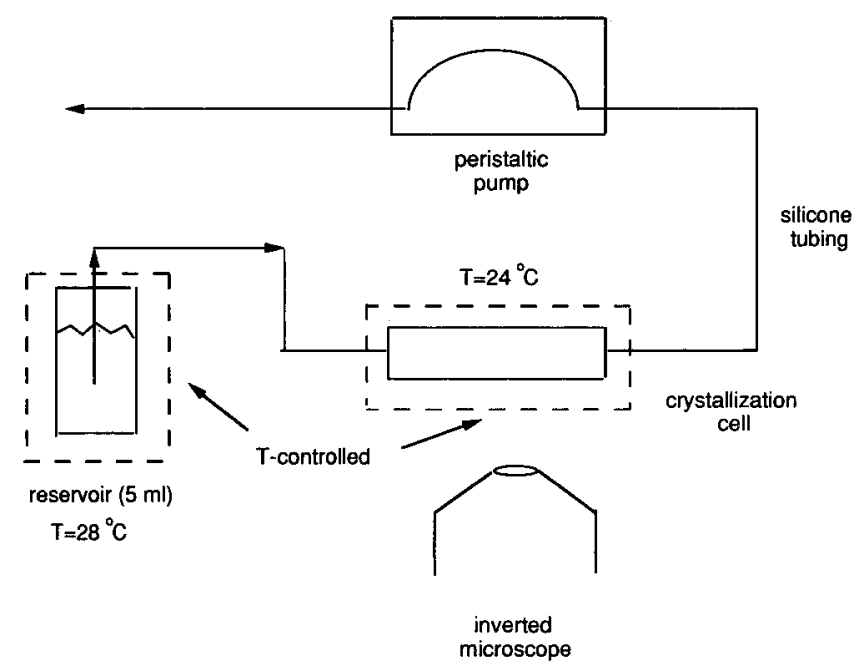

Figure 3-Schematic diagram of a flow system used to measure growth rates of crystal faces. The volume of the crystallization cell is 300 to $500 \mu \mathrm{L}$.

namically stable modification are based on the work of Davey, ${ }^{12-14,92}$ Leiserowitz,15,16 and others. ${ }^{40,93,94}$ The main features of these strategies are as follows:

(1) identification of the fastest growing faces of the stable crystalline modification,

(2) characterization of intermolecular interactions along the crystallographic direction with fastest growth,

(3) selection of additives that can be incorporated in the crystalline structure along this direction, and

(4) development of experimental methods to investigate the effectiveness of the additive to kinetically stabilize the metastable modification.

The selection of additives can be guided by molecular visualizations of the crystal structure based on geometric fits $^{13,95}$ or binding energy calculations. ${ }^{36,96}$

\section{Growth Mechanisms and Kinetics}

Once the nucleation step has been overcome, nuclei grow into macroscopic crystals. This stage of the crystallization process is known as crystal growth. The nucleation and growth processes compete for solute in terms of their respective dependence on supersaturation, and their relative rates will determine the crystal size distribution.

Crystal growth is governed by both internal and external factors. Internal factors such as the three-dimensional crystal structure and crystal defects will determine the nature and strength of the intermolecular interactions between the crystal surface and the solution, whereas external factors such as temperature, supersaturation, solvent, and the presence of impurities will affect the type of interactions at the solid-liquid interface.

Depending on the objectives and applications of growth rate measurements, the growth rate may be expressed as (1) overall linear growth rate which is the rate of change of the volume equivalent diameter with time, (2) linear growth rate of a face which is the rate of displacement of a crystal face in a direction perpendicular to the face, and (3) velocity, height, and spacing of growth steps spreading across a crystal surface. The linear growth rate of a face can be expressed in terms of the step velocity, the step height, and the step spacing. Techniques used for in-situ measurement of crystal growth rates as a function of supersaturation include (1) monitoring the crystal population by methods that measure particle size and number, ${ }^{97,98}$ (2) monitoring the growth rates of individual crystal faces by optical microscopy with the use of a flow cell system shown in Figure 3,39,95,99-101 and (3) monitoring the devel- opment of surface morphology at the molecular level by atomic force microscopy (AFM $)^{101-105}$ and interferometric microscopy. 37,38

The external shape or morphology of a crystal is a consequence of the relative growth rates of the faces and reveals the mol ecular events occurring at the crystal faceliquid interfaces during growth. Even when morphology does not play a significant role in quality control, studying it is essential to understand the kinetics of crystallization. ${ }^{106}$ The morphological importance of a face (surface area) is inversely related to its growth rate. Based on these concepts, one of the standard methods used for identifying solvents or dissolved additives that influence nucleation and growth is to investigate the relation between crystal morphology and growth conditions. ${ }^{13,95,107}$ A common approach is to compare the experimentally observed morphology with the theoretical morphology and to interpret differences on the basis of experimental growth parameters and assumptions of molecular modeling techniques. ${ }^{37-39}$

Batch-to-batch differences in crystal morphology may result from small but significant changes in growth conditions during the crystallization process. F or instance, the presence of a small amount of impurity can have a significant effect on the crystal habit. The most potent inhibitors of growth are impurities which are structurally similar to the host molecule (i.e., tailor-made additives and synthetic impurities). For example, the growth of L-alanine in the presence of hydrophobic L-amino acids at concentra-

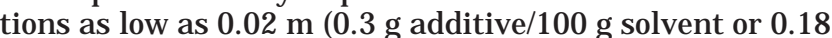
$\mathrm{g}$ additive/100 $\mathrm{g}$ L-alanine) results in a change in crystal morphology from prismatic to needle-shaped.95,101 The supersaturation at which crystal growth occurs can also significantly affect growth rates along different crystallographic axes and crystal habit. Growth of phenytoin crystals along the a crystallographic axis has a stronger dependence on supersaturation than growth along the $c$ axis. $^{39}$

The process of crystal growth consists of several stages through which growth units pass. These include (a) transport from the bulk solution to a site at the crystal surface, (b) adsorption of the growth unit onto the impingement site, or (c) diffusion from the impingement site to a growth site, and (d) incorporation into the crystal lattice. Desolvation can take place in steps b-d; however, the solvent has the possibility of being adsorbed.

Any one of the above steps may be ratelimiting depending on the growth conditions, such as the supersaturation, temperature, additives or solvent, and hydrodynamics of the system. Consequently, crystal growth mechanisms fall into two main categories: ${ }^{41-44}$ vol ume diffusion control and surface integration control, and the goals of crystal growth theories are to determine the source of steps and the ratecontrolling step for crystal growth.

As a crystal grows from a supersaturated solution, the solute concentration is depleted in the region of the crystal-solution interface. If diffusion of solute from the bulk solution to the crystal surface is rate limiting, growth is volume-diffusion controlled. A diffusion-controlled process is not applicable if there is no dependence of growth rate on hydrodynamic conditions such as flow rate or stirring rate. In practice it is difficult to completely eliminate volume diffusion resistance, and experimental growth kinetics can be misinterpreted as being purely surface-integration controlled when in fact diffusive resistance is still present. Garside has defined an effectiveness factor that is a measure of the relative importance of diffusion and surface integration as the rate-controlling factors for crystal growth. ${ }^{108}$

If incorporation into a crystal lattice is the slowest process, growth is surfaceintegration controlled. Many 
crystallization studies involving proteins, small organic electrolytes, and nonelectrolytes have reported growth controlled by surface integration. ${ }^{37-39,95,99-105}$ Depending on the roughness of the crystal surface, layer growth or continuous growth may result. If the crystal-solution interface at the molecular level is rough, there are many potential kink (growth) sites. In this case, continuous or normal growth models apply. Growth proceeds isotropically resulting in nonfaceted crystals and responds to very small gradients in the growth driving chemical potential (temperature, concentration, etc.). If the interface is smooth, growth proceeds through a layer growth model. Kink sites are only found on the edges of two-dimensional nuclei or steps; hence, surface diffusion and surface topography become more important.

Layer growth models describe the formation of steps by two mechanisms: screw dislocation and two-dimensional nucleation. Details of the derivation of these models can be found in the literature. ${ }^{42,44,109-111}$

The spiral growth mechanism or screw dislocation model was first described by Burton, Cabrera, and Frank (BCF). ${ }^{112}$ Bennema111,113,114 modified this theory for crystals growing from aqueous solution. Flat crystal surfaces have a high energy barrier for nucleation at low supersaturations, and the presence of screw dislocations provides a source of steps for the addition of growth units in an infinite sequence of equidistant and parallel steps. In the simplest case, these steps will devel op as a spiral which rotates about its axis during growth.

Two-dimensional nucleation requires the formation of clusters above a critical size for growth of layers. The same concepts discussed in the nucleation section apply to the nucleation of a two-dimensional cluster above a critical size. This model accounts for crystal growth at high supersaturations. These models were developed by Volmer ${ }^{115}$ and Stranski. ${ }^{116}$ Variations in the mode that nuclei spread after being formed lead to the various two-dimensional nucle ation models: mononuclear, polynuclear, and birth and spread.

Each crystal growth model describes the growth rate dependence on supersaturation, temperature, and face area. Although the various models can be used to identify the growth mechanism by fitting the various equations to the experimentally measured face-growth rate dependence on supersaturation, it may be difficult to discriminate between models. ${ }^{39,101,117}$ This approach is often combined with observations of surface topography to confirm the growth mechanism.

Surface mi crotopographic observations can reveal growth mechanisms of crystal faces under different growth conditions, as shown for human insulin crystals in Figure 4. Monitoring the development of surface topography and transport processes during crystal growth will reveal events that are not evident from morphology studies. Interactions of growth units and additives with different crystal planes exposed on a surface may be deduced from the shape of two-dimensional nuclei, and the kinetic anisotropy of the growth steps al ong crystallographic directions. These techniques have been successfully applied for identifying the crystal growth mechanisms and kinetics of small molecules $37,38,101$ and proteins. ${ }^{102-105,118}$

\section{Solution-Mediated Transformations}

Knowledge of the propensity of a metastable solid phase to dissolve in a liquid phase from which a stable solid phase nucleates and grows is crucial in many stages of pharmaceutical development, because pharmaceutical solids are designed to be dissolved and to come in contact with solvents since the early stages of development (isolated by

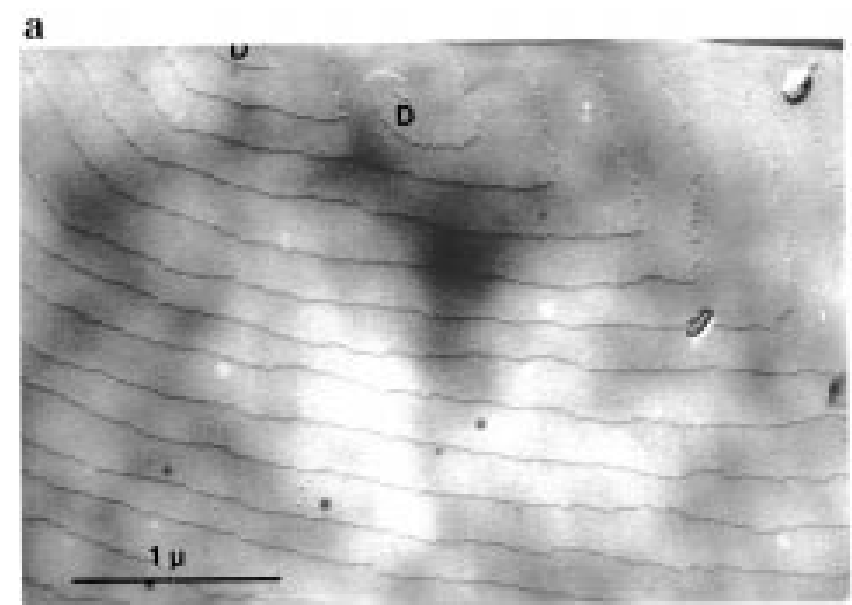

b

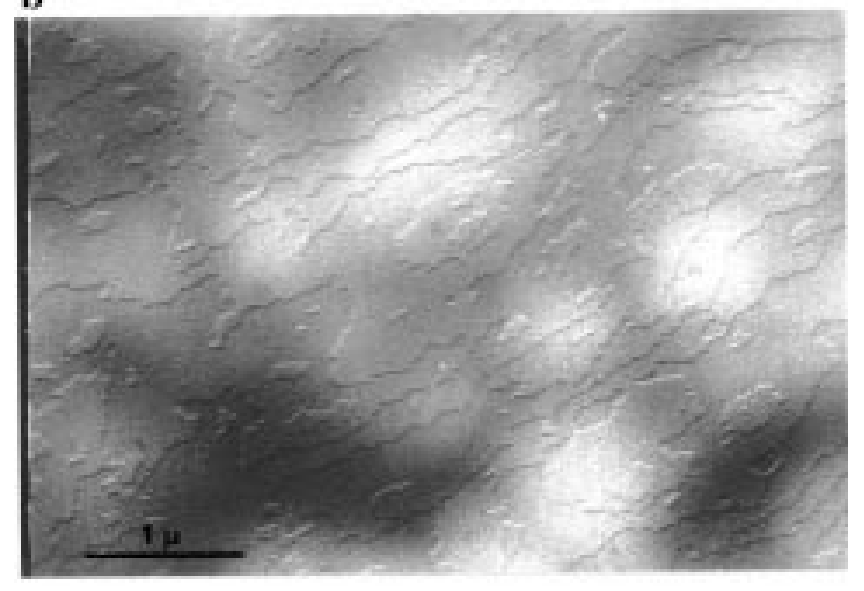

Figure 4-Micrographs of human insulin crystals showing that steps arise by different mechanisms depending on supersaturation. (a) At low supersaturation, steps are created by screw dislocations, D. (b) At high supersaturation, $c / s$ $>10$, steps occur at the edges of islands as a consequence of two-dimensional nucleation.

crystallization from solution) and during processing (wet granulation, spray-drying, freeze-drying, etc.). Given that the sudden disappearance or appearance of a crystalline modification can threaten process development, characterization of the kinetics and mechanisms of solvent-mediated transformations is of practical importance. It will provide answers to questions such as the following: What is the relation between processing conditions and the solid-state modification manufactured? Is there a correlation between dissolution conditions, solid phase(s) dissolving, and concentration of drug dissolved?

The importance of phase transition kinetics, molecular interpretations, and process implications are emphasized by several investigators. ${ }^{5-9,12-14,119}$ Cardew and Davey ${ }^{5}$ developed a theoretical framework to investigate solventmediated transformations in terms of dissolution kinetics of one phase and growth of a second phase. The model represents the time development of the supersaturation with respect to the stable phase, or solute concentration in solution, and solid phase composition during the transformation. The experimental approach involves saturating the solution with respect to the metastable phase under consideration and to monitor both solution concentration and solid phase composition in the presence of the metastable phase, under constant external conditions. More useful information is obtained from the concentration or supersaturation profiles than from the solid phase composition profiles with time. Since the former is related to the 


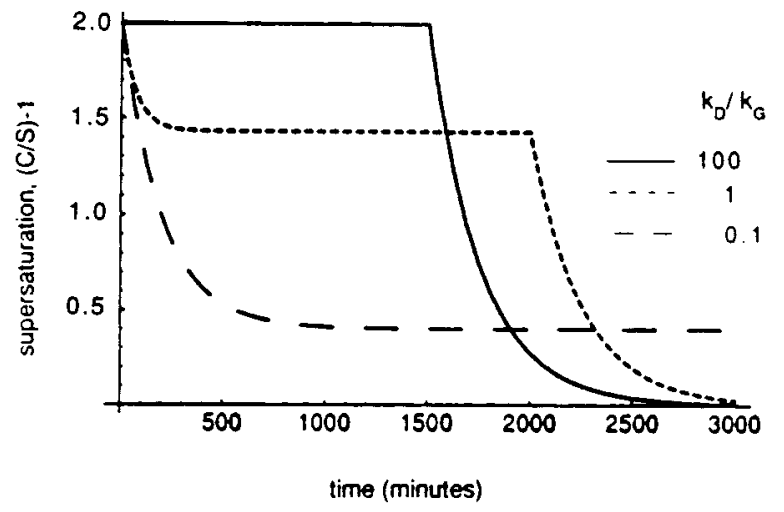

Figure 5-Numerical simulation of the supersaturation-time profiles, as a function of the relative rates of dissolution and crystallization during a solutionmediated transformation. Generated from a kinetic model developed by Cardew and Davey, ${ }^{5}$ assuming that both dissolution and growth rates are linearly dependent on their respective driving forces.

Table 3-Information on Crystallization Kinetics and Mechanisms Provided by Various Experimental Techniques

\begin{tabular}{|c|c|}
\hline techniques & information \\
\hline $\begin{array}{l}\text { optical microscopy } \\
\text { (inverted microscope) }\end{array}$ & $\begin{array}{l}\text { - study crystallization processes in situ } \\
\text { - monitor transformations in suspensions } \\
\text { - determine transformation times } \\
\text { - screen and characterize additive/solvent interactions } \\
\text { with specific crystal faces } \\
\text { - identify nucleation mechanisms } \\
\text { - measure crystal growth rates }\end{array}$ \\
\hline electron microscopy & $\begin{array}{l}\text { - characterize additive/solvent interactions with specific } \\
\text { crystal faces } \\
\text { - identify nucleation and growth mechanisms } \\
\text { - measure crystal growth rates }\end{array}$ \\
\hline $\begin{array}{l}\text { atomic force microscopy } \\
\text { interferometric microscopy }\end{array}$ & $\begin{array}{l}\text { - study crystallization processes in situ } \\
\text { - examine surface topography } \\
\text { - identify nucleation and growth mechanisms } \\
\text { - measure crystal growth rates }\end{array}$ \\
\hline Raman spectroscopy & $\begin{array}{l}\text { - monitor molecular association processes that direct } \\
\text { nucleation and crystal growth }\end{array}$ \\
\hline $\begin{array}{l}\text { spectrophotometry } \\
\text { chromatography }\end{array}$ & $\begin{array}{l}\text { - monitor concentration of solute in solution and } \\
\text { supersaturation }\end{array}$ \\
\hline $\begin{array}{l}\text { diffraction } \\
\text { calorimetry } \\
\text { spectroscopy }\end{array}$ & - monitor solid phase composition \\
\hline
\end{tabular}

driving forces that regulate the transformation rate and can be used to identify the rate-controlling process: dissolution or growth. A calculated supersaturation profile with growth-limited and dissolution-limited regimes is shown in Figure 5, for the case in which dissolution and growth are linearly dependent on supersaturation. ${ }^{5,6}$ Experimental studies of the phase transitions of organic crystals have shown this model to be applicable to explain the solution-mediated transformation kinetics of polymorphs $s^{5,6,119}$ and solvates, ${ }^{8}$ and to be applicable to process development. ${ }^{119}$ Some useful experimental techniques for studying small and large scale crystallization and solutionmediated transformations are summarized in Table 3.

\section{Summary}

In this review we have presented the significance of crystallization mechanisms and kinetics in directing crystallization pathways. Given the recent advances in computational and analytical techniques that provide access to the molecular events that direct nucleation and crystal growth, there is no reason for the development of ill-defined crystallization processes even when the desired product is obtained. Understanding the thermodynamic and kinetic behavior of the system is vital for the design of reliable processes; thus, at least a holistic approach that identifies the relevant experimental parameters is required. While the objectives of formulation or process development may not explicitly include interpretations at the mol ecular level, the information concealed in the interfaces present during the process, the structure of the solution, and the solids harvested (crystal structure, morphology, particle size and number) can be significant in the identification of nucleation and growth mechanisms, and will reveal molecular events associated with crystallization. Although the significance of crystallization mechanisms and kinetics has been underestimated in the pharmaceutical industry, we hope that the recent developments in crystallization engineering and techniques will inspire interest in the application of this field to pharmaceutical systems.

\section{References and Notes}

1. Marshall, P. V.; York, P. Crystallization Solvent Induced Solid State and Particulate Modifications of Nitrofurantoin. Int. J. Pharm. 1989, 55, 257-263.

2. Ludlambrown, I.; York, P. The Crystalline Modification of Succinic Acid by Variations in Crystallization Conditions. J . Phys. D: Appl. Phys. 1993, 26, B60-B65.

3. Byrn, S. R.; Pfeiffer, R. R.; Stephenson, G.; Grant, D. J . W.; Gleason, W. B. Solid-State Pharmaceutical Chemistry. Chem. Mater. 1994, 6, 1148-1158.

4. Byrn, S.; Pfeiffer, R.; Ganey, M.; Hoiberg, C.; Poochikian, G. Pharmaceutical Solids: A Strategic Approach to Regulatory Considerations. Pharm. Res. 1995, 12, 945-954.

5. Cardew, P. T.; Davey, R. J . The Kinetics of Solvent-Mediated Phase Transformations. Proc. R. Soc. London 1985, A 398, 415-428.

6. Davey, R. J.; Cardew, P. T.; Mcewan, D.; Sadler, D. E. Rate Controlling Processes in Solvent-M ediated Phase Transformations. J. Cryst. Growth 1986, 79, 648-653.

7. Boylan, J. C.; Swarbrick, J . Crystallization and theProperties of Crystals; Rodríguez-Hornedo, N., Ed.; Marcel Dekker Inc.: New York, 1991; Vol. 3, pp pp 399-434.

8. Rodríguez-Hornedo, N.: Lechuga-Ballesteros, D.; Wu, H. J. Phase Transition and Heterogeneous Epitaxial Nucleation of Hydrated and Anhydrous Theophylline Crystals. Int. J. Pharm. 1992, 85, 149-162.

9. Boylan, J . C.; Swarbrick, J . Hydrates; Morris, K., RodríguezHornedo, N., Eds.; 1993; Vol. 7, pp 393-440.

10. Zhu, H. J.; Grant, D. J . W. Influence of Water Activity in Organic Solvent Plus Water Mixtures on the Nature of the Crystallizing Drug Phase. 2. Ampicillin. Int. J . Pharm. 1996, $139,33-43$.

11. Zhu, H. J .; Yuen, C. M.; Grant, D. J . W. Influence of Water Activity in Organic Solvent Plus Water Mixtures on the Nature of the Crystallizing Drug Phase. 1. Theophylline. Int. J . Pharm. 1996, 135, 151-160.

12. Davey, R. J .; Black, S. N.; Goodwin, A. D.; Mackerron, D.; Maginn, S. J .; Miller, E. J. Crystallisation in Polymer Films: Control of Morphology and Kinetics of an Organic Dye in a Polysilicone Matrix. J . Mater. Chem. 1997, 7, 237241.

13. Davey, R. J .; Blagden, N.; Potts, G. D.; Docherty, R. Polymorphism in Molecular Crystals: Stabilization of a Metastable Form by Conformational Mimicry. J . Am. Chem. Soc. 1997, 119, 1767-1772.

14. Blagden, N.; Davey, R. J .; Lieberman, H. F.; Williams, L.; Payne, R.; Roberts, R.; Rowe, R.; Docherty, R. Crystal Chemistry and Solvent Effects in Polymorphic Systems - Sulfathiazole. J. Chem. Soc., Faraday Trans. 1998, 94 1035-1044.

15. Weissbuch, I.; Leisorowitz, L.; Lahav, M. Tailor-Made and Charge-Transfer Auxiliaries for the Control of the Crystal Polymorphism of Glycine. Adv. Mat. 1994, 6, 952-956.

16. Weissbuch, I.; Zbaida, D.; Addadi, L.; Leiserowitz, L.; Lahav M. Design of Polymeric Inhibitors for the Control of Crystal Polymorphism - Induced Enantiomeric Resolution of Race mic Histidine by Crystallization at $25^{\circ} \mathrm{C}$. J . Am. Chem. Soc. 1987, 109, 1869-1871.

17. Dunitz, J . D.; Bernstein, J . Disappearing Polymorphs. Acc. Chem. Res. 1995, 28, 193-200. 
18. Abbott Laboratories. Letter to health care providers. J uly 1998. http://www.fda.gov/medwatch/safety/1998/norvir.htm accessed March 1999.

19. Nichols, G.; Frampton, C. S. Physicochemical Characterization of the Orthorhombic Polymorph of Paracetamol Crystallized From Solution. J . Pharm. Sci. 1998, 87, 684-692.

20. Haisa, M.; Kashino, S.; Maeda, H. The Orthorhombic Form of P-Hydroxyacetanilide. Acta Crystallogr. 1974, B30, 25102512.

21. Food and Drug Administration Guideline for Submitting Supporting Documentation in Drug Applications for the Manufacture of Drug Substances; FDA Center for Drug Evaluation and Research: Office of Drug Evaluation: Rockville, MD, 1987

22. Desiraju, G. R. Supramolecular Synthons in Crystal Engineering - A N ew Organic-Synthesis. Angew. Chem., Int. Ed. Engl. 1995, 34, 2311-2327.

23. Desiraju, G. R. Crystal Gazing: Structure Prediction and Polymorphism. Science 1997, 278, 404-405.

24. Desiraju, G. R. Designer Crystals: Intermolecular Interactions, Network Structures and Supramolecular Synthons. Chem. Commun. 1997, 1475-1482.

25. Gavezzotti, A. Are Crystal Structures Predictable. Acc. Chem Res. 1994, 27, 309-314.

26. Gavezzotti, A. Computer Simulations of Organic Solids and Their Liquid-State Precursors. Faraday Discuss. 1997, 106, 63-77.

27. Gavezzotti, A.; Filippini, G. Polymorphic Forms of Organic Crystals at Room Conditions - Thermodynamic and Structural Implications. J. Am. Chem. Soc. 1995, 117, 1229912305.

28. Gavezzotti, A.; Filippini, G. Computer Prediction of Organic Crystal Structures Using Partial X-ray Diffraction Data. J . Am. Chem. Soc. 1996, 118, 7153-7157.

29. Gavezzotti, A.; Filippini, G. Self-Organization of Small Organic Molecules in Liquids, Solutions and Crystals: Static and Dynamic Calculations. Chem. Commun 1998, 287-294.

30. Blagden, N.; Davey, R. J .; Lieberman, H. F.; Williams, L.; Payne, R.; Roberts, R.; Rowe, R.; Docherty, R. Crystal Chemistry and Solvent Effects in Polymorphic Systems: Sulfathiazole. J . Chem. Soc., Faraday Trans. 1998, 94, 19191919.

31. Weissbuch, I.; Popovitzbiro, R.; Lahav, M.; Leiserowitz, L. Understanding and Control of Nucleation, Growth, Habit, Dissolution and Structure of 2-Dimensional and 3-Dimensional Crystals Using Tailor-Made Auxiliaries. Acta Crystallogr., Sect. B: Struct. Sci. 1995, 51, 115-148.

32. Leiserowitz, L.; Addadi, L.; Lahav, M. Macroscopic Phenomena in Crystals and Molecular Shape. J. Mol. Graph. 1989, 7, 95-95.

33. Weissbuch, I.; Addadi, L.; Lahav, M.; Leiserowitz, L. Molecular Recognition at Crystal Interfaces. Science 1991, 253, 637-645.

34. Gallagher, H. G.; Roberts, K. J .; Sherwood, J . N.; Smith, L. A. A Theoretical Examination of the Molecular Packing, Intermolecular Bonding and Crystal Morphology of 2,4,6Trinitrotoluene in Relation to Polymorphic Structural Stability. J . Mater. Chem. 1997, 7, 229-235.

35. Clydesdale, G.; Roberts, K.J .; Telfer, G. B.; Grant, D. J . W Modeling the Crystal Morphology of Alpha-Llactose Monohydrate. J. Pharm. Sci. 1997, 86, 135-141.

36. Hendriksen, B. A.; Grant, D. J . W.; Meenan, P.; Green, D. A. Crystallisation of Paracetamol (Acetaminophen) in the Presence of Structurally Related Substances. J . Cryst. Growth 1998, 183, 629-640.

37. Shekunov, B. Y.; Latham, R. Growth Anisotropy of NMethylurea Crystals in Methanol. J. Phys. Chem. A 1996 100, 5464-5469.

38. Shekunov, B. Y.; Grant, D. J. W. In Situ Optical Interferometric Studies of the Growth and Dissolution Behavior of Paracetamol (Acetaminophen) 0.1. Growth Kinetics. J . Phys. Chem. B 1997, 101, 3973-3979.

39. Zipp, G. L.; Rodríguez-Hornedo, N. Growth Mechanism and Morphology of Phenytoin and Their Relationship With Crystallographic Structure. J . Phys. D: Appl. Phys. 1993, 26, B48-B55.

40. Khoshkhoo, S.; Anwar, J . Study of the Effect of Solvent on the Morphology of Crystals Using Molecular Simulation: Application to Alpha-Resorcinol and N-N-Octyl-D-Gluconamide. J. Chem. Soc., Faraday Trans. 1996, 92, 1023-1025.

41. Nyvlt, J.; Söhnel, O.; Matuchová, M.; Broul, M. TheKinetics of Industrial Crystallization; Elsevier: New York, 1985

42. Mullin, J. W. Crystallization; Butterworth-Heinemann Ltd.: Oxford, 1993.

43. Söhnel, O.; Garside, J. Precipitation: Basic Principles and Industrial Applications; Butterworth-Heinemann Ltd: Oxford, 1992
44. Myerson, A. S. Handbook of Industrial Crystallization; Butterworth-Heinemann Ltd: Oxford, 1993.

45. Mersmann, A. Crystallization Technology Handbook; M. Dekker: New York, 1995

46. Zettlemoyer, A. C. Nucleation; Marcel Dekker: New York, 1969.

47. Perepezko, J. H. Nucleation Reactions In Undercooled Liquids. Mater. Sci. Eng. A 1994, 178, 105-111.

48. Perepezko, J. H. Kinetic Processes in Undercooled Melts. Mater. Sci. Eng. A 1997, 226, 374-382.

49. Gibbs, J. W. Collected Works, Vol. I, Thermodynamics; Yale University Press: New Haven, 1948.

50. Volmer, M. Kinetic Der Phasenbildung; Steinkopff, Leipzig: 1939.

51. Becker, R.; Döring, W. Kinetische Behandlung Der Keimbildung in Übersättigen Dämpfen. Ann. Physik (Leipzig) 1935, 24, 719-752.

52. Turnbull, D.; Fisher, J . C. Rate of Nucleation in Condensed Systems. J . Chem. Phys. 1949, 17, 71-73.

53. Boistelle, R.; Astier, J. P. Crystallization Mechanisms in Solution. J . Cryst. Growth 1988, 90, 14-30.

54. Hendriksen, B. A.; Grant, D. J . W. The Effect of Structurally Related Substances on the Nucleation Kinetics of Paracetamol (Acetaminophen). J . Cryst. Growth 1995, 156, 252-260.

55. Garti, N.; Tibika, F. Habit Modifications of Nitrofurantoin Crystallized From Formic Acid Mixtures. Drug Dev. Ind. Pharm. 1980, 6, 379-398.

56. Ostwald, W. Studien Über Die Bildung Und Umwandlung Fester Körper. Zieitschrigt Für Physikalische Chemie 1897, 22, 289-330.

57. Pellett, M. A.; Castellano, S.; Hadgraft, J .; Davis, A. F. The Penetration of Supersaturated Sol utions of Piroxicam Across Silicone Membranes and Human Skin in Vitro. J . Controlled Rel ease 1997, 46, 205-214.

58. Katzhendler, I.; Azoury, R.; Friedman, M. Crystalline Properties of Carbamazepine in Sustained Release Hydrophilic Matrix Tablets Based on Hydroxypropylmethylcellulose. J . Controlled Release 1998, 54, 69-85.

59. Davey, R. J .; Hilton, A. M.; Garside, J . Crystallization From Oil in Water Emulsions: Particle Synthesis and Purification of Molecular Materials. Chem. Eng. Res. Des. 1997, 75, 245251

60. Davey, R.J .; Garside, J .; Hilton, A. M.; Mcewan, D.; Morrison, J. W. Purification of Molecular Mixtures Below the Eutectic by Emulsion Crystallization. Nature 1995, 375 , 664-666.

61. Davey, R. J .; Garside, J .; Hilton, A. M.; Mcewan, D.; Morrison, J. W. Emulsion Solidification of Meta-Chloronitrobenzene: Purification and Crystallisation. J . Cryst. Growth 1996, 166, 971-975.

62. Turnbull, D. Under What Conditions Can a Glass Be Formed? Contemp. Phys. 1969, 10, 473-488.

63. Murase, N.; Franks, F. Salt Precipitation During the Freeze Concentration of Phosphate Buffer Solutions. Biophys. Chem. 1989, 34, 293-300.

64. Murase, N.; Echlin, P.; Franks, F. The Structural States of Freeze-Concentrated and Freeze-Dried Phosphates Studied by Scanning Electron-Microscopy and Differential Scanning Calorimetry. Cryobiology 1991, 28, 364-375.

65. Cavatur, R. K.; Suryanarayanan, R. Characterization of Frozen Aqueous Solutions by Low-Temperature X-ray Powder Diffractometry. Pharm. Res. 1998, 15, 194-199.

66. Gómez, G. Crystallization-Related pH Changes During Freezing of Sodium Phosphate Buffer Solutions; Ph.D. Thesis, University of Michigan, Ann Arbor, MI, 1995.

67. Mullin, J. W.; Leci, C. L. Some Nucleation Characteristics of Aqueous Citric Acid Solutions. J . Cryst. Growth 1969, 5, 75-76.

68. Franks, F.; Mathias, S. F.; Trafford, K. The Nucleation of I ce in Undercooled Water and Aqueous Polymer Solutions. Colloids Surfaces 1984, 11, 275-285.

69. Fletcher, N. H. Nucleation by Crystalline Particles. J . Chem. Phys. 1963, 38, 237-240.

70. Carter, P. W.; Ward, M. D. Topographically Directed Nucleation of Organic-Crystals on Molecular Single-Crystal Substrates. J. Am. Chem. Soc. 1993, 115, 11521-11535.

71. Ward, M. D. Organic Crystal Surfaces: Structure, Properties and Reactivity. Curr. Opin. Colloid Interface Sci. 1997, 2 $51-64$.

72. Carter, P. W.; Ward, M. D. Directing Polymorph Selectivity During Nucleation of Anthranilic Acid on Molecular Substrates. J. Am. Chem. Soc. 1994, 116, 769-770.

73. Black, S. N.; Bromley, L. A.; Cottier, D.; Davey, R. J .; Dobbs, B.; Rout, J. E. Interactions at the Organic Inorganic Interface - Binding Motifs for Phosphonates at the Surface of Barite Crystals. J . Chem. Soc., Faraday Trans. 1991, 87, 34093414 
74. Bonafede, S.J .; Ward, M. D. Selective Nucleation and Growth of an Organic Polymorph by Ledge-Directed Epitaxy on a Molecular-Crystal Substrate. J . Am. Chem. Soc. 1995, 117, 7853-7861.

75. Davey, R. J .; Maginn, S. J .; Andrews, S. J .; Black, S. N.; Buckley, A. M.; Cottier, D.; Dempsey, P.; Plowman, R.; Rout, J. E.; Stanley, D. R.; Taylor, A. Morphology and Polymorphism of Terephthalic Acid. Mol. Cryst. Liq. Cryst. Sci. Technol., Sect. A: Crystals And Liquid Crystals 1994, 242, 79-90.

76. De Smidt, J. G.; Fokkens, J. G.; Grijseels, H.; Crommelin, D. J. A. Dissolution of Theophylline Monohydrate and Anhydrous Theophyline in Buffer Solutions. J. Pharm. Sci. 1986, 75, 497-501.

77. Luhtala, S.; Kahela, P.; Kristoffersson E. Effect of BenzaIkonium Chloride on Crystal Growth and Aqueous Solubility of Carbamazepine. Acta Pharm. Fenn. 1990, 99, 59-68.

78. Luhtala, S. Effect of Sodium Lauryl Sulfate and Polysorbate 80 on Crystal Growth and Aqueous Solubility of Carbamazepine. Acta Pharm. Nord. 1992, 4, 85-90.

79. Murphy, D. The Solvent-M ediated Phase Transformation of Carbamazepine and the Influence of Surfactants on the Nucleation Mechanism and Crystal Morphology; Ph.D. Thesis, University of Michigan, Ann Arbor, MI, 1997.

80. Kadima, W.; McPherson, A.; Dunn, M. F.; J urnak, F. Pre crystallization Aggregation of Insulin by Dynamic LightScattering and Comparison With Canavalin. J . Cryst. Growth 1991, 110, 188-194.

81. Georgalis, Y.; U mbach, P.; Raptis, J .; Saenger, W. Lysozyme Aggregation Studied by Light Scattering 0.1 . I nfluence of Concentration and Nature of Electrolytes. Acta Crystallogr., Sect. D: Biol. Crystallogr. 1997, 53, 691-702.

82. Georgalis, Y.; U mbach, P.; Raptis, J .; Saenger, W. Lysozyme Aggregation Studied by Light Scattering. 2. Variations of Protein Concentration. Acta Crystallogr., Sect. D: Biol. Crystallogr. 1997, 53, 703-712.

83. Peters, R.; Georgalis, Y.; Saenger, W. Accessing Lysozyme Nucleation With a Novel Dynamic Light Scattering Detector. Acta Crystallogr., Sect. D: Biol. Crystallogr. 1998, 54, 873877.

84. Rosenberger, F.; Vekilov, P. G.; Muschol, M.; Thomas, B. R. Nucleation and Crystallization of Globular Proteins - What We Know and What Is Missing. J . Cryst. Growth 1996, 168, $1-27$.

85. Cerreta, M. K.: Berglund, K. A. The Structure of AqueousSolutions of Some Dihydrogen Ortho-Phosphates by Laser Raman-Spectroscopy. J. Cryst. Growth 1987, 84, 577-588.

86. Yedur, S. K.: Berglund, K. A. Use of Fluorescence Spectroscopy in Concentration and Supersaturation Measurements in Citric Acid Solutions. Appl. Spectrosc. 1996, 50, 866-870.

87. Rasimas, J . P.; Berglund, K. A.; Blanchard, G. J . A Molecular Lock and Key Approach to Detecting Solution Phase SelfAssembly. A Fluorescence and Absorption Study of Carminic Acid in Aqueous Glucose Solutions. J . Phys. Chem. 1996, 100, 7220-7229.

88. Dunuwila, D. D.; Berglund, K. A. Identification of Infrared Spectral Features Related to Solution Structure for Utilization in Solubility and Supersaturation Measurements. Org. Proc. Res. Dev. 1997, 1, 350-354.

89. Boistelle, R.; López-Valero, I. Growth Units and Nucleation: the Case of Calcium Phosphates. J . Cryst. Growth 1990, 102, 609-617.

90. Gidal evitz, D.; Feidenhansl, R.; Matlis, S.; Smilgies, D. M.; Christensen, M. J .; Leiserowitz, L. Monitoring In Situ Growth and Dissolution of Molecular Crystals: Towards Determination of the Growth Units. Angew. Chem., Int. Ed. Engl. 1997, 36, 955-959.

91. Khoshkhoo, S.; Anwar, J . Crystallization of Polymorphs The Effect of Solvent. J . Phys. D: Appl. Phys. 1993, 26, B90B93.

92. Black, S. N.: Davey, R. J : Halcrow, M. The Kinetics of Crystal-Crowth in the Presence of Tailor-Made Additives. J . Cryst. Growth 1986, 79, 765-774.

93. Chen, B. D.; Garside, J .; Davey, R. J .; Maginn, S. J.; Matsuoka, M. Growth of M-Chloronitrobenzene Crystals in the Presence of Tailor-Made Additives - Assignment of the Polar Axes From Morphological Calculations. J . Phys. Chem. 1994, 98, 3215-3221.

94. Garti, N.; Zour, H. The Effect of Surfactants on the Crystallization and Polymorphic Transformation of Glutamic Acid. J . Cryst. Growth 1997, 172, 486-498.

95. Lechuga-Ballesteros, D.; Rodríguez-Hornedo, N. Effects of Molecular Structure and Growth Kinetics on the Morphology of L-Alanine Crystals. Int. J . Pharm. 1995, 115, 151-160.

96. Etter, M. C. Hydrogen-Bonds As Design Elements in Organic Chemistry. J . Phys. Chem. 1991, 95, 4601-4610.
97. Zipp, G. L.; Rodríguez-Hornedo, N. Phenytoin Crystal Growth Rates in the Presence of Phosphate and Chloride Ions. J . Cryst. Growth 1992, 123, 247-254.

98. Lechuga-Ballesteros, D.; Rodríguez-Hornedo, N. The Relation Between Adsorption of Additives and Crystal Growth Rate of L-Alanine. J. Colloid Interface Sci. 1993, 157, 147-153.

99. Li, L.; Rodríguez-Hornedo, N. Growth Kinetics and Mechanisms of Glycine Crystals. J . Cryst. Growth 1992, 121, 3338.

100. Zipp, G. L.; Rodríguez-Hornedo, N. The Mechanism of Phenytoin Crystal-Growth. Int. J. Pharm. 1993, 98, 189201.

101. Lechuga-Ballesteros, D.; Rodríguez-Hornedo, N. The Influence of Additives on the Growth Kinetics and Mechanism of L-Alanine Crystals. Int. J . Pharm. 1995, 115, 139-149.

102. Yip, C. M.; Ward, M. D. Atomic Force Microscopy of Insulin Single Crystals: Direct Visualization of Molecules and Crystal Growth. Biophys. J . 1996, 71, 1071-1078.

103. Malkin, A. J .; Kuznetsov, Y. G.; Glantz, W.; McPherson, A. Atomic F orce Microscopy Studies of Surface M orphology and Growth Kinetics in Thaumatin Crystallization. J. Phys. Chem. 1996, 100, 11736-11743.

104. Yip, C. M.; Brader, M. L.; Defelippis, M. R.; Ward, M. D Atomic F orce Microscopy of Crystalline Insulins: The Influence of Sequence Variation on Crystallization and Interfacial Structure. Biophys. J . 1998, 74, 2199-2209.

105. Kuznetsov, Y. G.; Malkin, A. J .; McPherson, A. Atomic Force Microscopy Studies of Phase Separations in Macromolecular Systems. Phys. Rev. B: Condens. Matter 1998, 58, 60976103.

106. Sunagawa, I. Morphol ogy of Crystals; Terra Scientific Publishing Company: Tokyo, J apan, 1987.

107. Li, L.; Lechuga-Ballesteros, D.; Szkudlarek, B. A.; RodríguezHornedo, N. The Effect of Additives on Glycine Crystal Growth Kinetics. J . Colloid Interface Sci. 1994, 168, 8-14.

108. Garside, J. The Concept of Effectiveness Factors in Crystal Growth From Solution. Chem. Eng. Sci. 1971, 26, 14251431.

109. Chernov, A. A. Formation of Crystals in Solution. Contemp. Phys. 1989, 30, 251-276.

110. Chernov, A. A. Modern Crystallography III: Crystal Growth; Springer-Verlag: Berlin, 1980.

111. Bennema, P. Spiral Growth and Surface Roughening: Development Since Burton, Cabrera and Frank. J. Cryst. Growth 1984, 69, 182-197.

112. Burton, W. K.; Cabrera, N.; Frank, F. C. The Growth of Crystals and the Equilibrium Structure of Their Surfaces. Philos. Trans. 1951, A243, 299-358.

113. Bennema, P. Analysis of Crystal Growth Models for Slightly Supersaturated Solutions. J. Cryst. Growth 1967, 1, 225232.

114. Bennema, P. Interpretation of the Relation of the Rate of Crystal Growth and Low Supersaturation. J . Cryst. Growth 1967, 1, 278-287.

115. Volmer, M. Crystal Growth. Z. Physik. 1922, 9, 193.

116. Stranski, I. N. Zur Theorie Der Kristallwachstums. Z. Phys. Chem. 1928, 136, 259-278.

117. Garside, J .; J anssen-Van Rosmalen, R.; Bennema, P. Verification of Crystal Growth Rate Equations. J . Cryst. Growth 1975, 29, 353-366.

118. Durbin, S. D.; Feher, G. Studies of Crystal Growth Mechanisms of Proteins by Electron Microscopy. J . Mol. Biol . 1990, $212,763-774$.

119. Nass, K. K. Process I mplications of Polymorphism in Organic Compounds. AIChE 1991, 284, 72-81.

\section{Acknowledgments}

The authors acknowledge financial support from the National Science Foundation, Valteich Research Award from the University of Michigan College of Pharmacy, Elli Lilly and Company, and Dupont Pharma.

\section{J $\mathrm{S} 980490 \mathrm{H}$}

